unknown whether sensing occurs directly or via intermediary proteins.

The cryopyrin inflammasome is implicated in other autoinflammatory diseases as well as gouty inflammation. Gain-of-function mutations in cryopyrin lead to childhood autoinflammatory diseases characterized by constitutive processing of IL-1 $\beta$, recurrent fever and inflammation. These symptoms are improved by treatments designed to block IL-1 $1 \beta$, and it is anticipated that similar treatments could benefit patients with gout and pseudogout. Moreover, these results seem to provide an explanation for the capacity of colchicine to control gouty inflammation.

Rachel Murphy

Original article Martinon F et al. (2006) Gout-associated uric acid crystals activate the NAPL3 inflammasome. Nature [doi:10.1038/nature04516]

\section{Congestive heart failure contributes to excess deaths in rheumatoid arthritis}

Mortality in patients with rheumatoid arthritis (RA) is higher than in the general population. Deaths caused directly by RA are rare; the additional deaths must, therefore, result from other conditions. It is not known, however, to what extent individual comorbidities contribute to excess mortality in patients with RA.

Researchers at the Mayo Clinic investigated the contributions of ischemic heart disease and of congestive heart failure (CHF) to mortality in patients with $R A$, in a retrospective study of 603 individuals diagnosed between 1955 and 1995, matched with a non-RA control population. There were 345 deaths in the RA group compared with 222 in the control group. Statistically, there was no difference in the incidence of myocardial infarction or ischemic heart disease between the groups, but the incidence of $\mathrm{CHF}$ was significantly higher in the RA group (37.1\% versus $27.7 \%, P<0.001)$. As the death rate for patients with heart conditions was similar in both groups, it seems that the increased incidence of $\mathrm{CHF}$ in the RA population is primarily responsible for the excess deaths attributable to $\mathrm{CHF}$ in patients with RA.

The reasons for the increased incidence of CHF observed in RA are unclear. It has been reported that high levels of systemic markers of inflammation are predictive of $\mathrm{CHF}$, and C-reactive protein has been found to be a risk factor for the condition. Pericardial and valve disease, which are possible complications of $R A$, and potential cardiotoxic effects of RA drugs, could also be implicated.

Jim Casey

Original article Nicola PJ et al. (2006) Contribution of congestive heart failure and ischemic heart disease to excess mortality in rheumatoid arthritis. Arthritis Rheum 54: 60-67

\section{Some treatments for RA affect the immune response to pneumococcal vaccine}

Infections are an important cause of morbidity and death in patients with rheumatoid arthritis (RA), perhaps because of disease-associated immune dysfunction and treatment with immunosuppressive drugs. Severe Streptococcus pneumoniae infections can occur in patients with RA receiving TNF blockers; Swedish researchers, therefore, administered a 23-valent pneumococcal vaccine to patients receiving various therapies for RA. They compared the immune responses of those treated with methotrexate, TNF blockers (with or without other diseasemodifying antirheumatic drugs), or both, with the responses of healthy control individuals.

Neither anti-TNF therapy nor prednisolone affected the immune responses of patients, but a marked impairment occurred with methotrexate treatment. Patients treated with both TNF blockers and methotrexate had a lower response to the vaccine than those treated with anti-TNF agents alone, and patients receiving methotrexate alone had the lowest immune response.

The authors postulate that immunosuppression might be a mode of action of methotrexate in RA treatment, and that anti-TNF agents might enhance the immune response, accounting for the difference between patients treated with methotrexate alone and those who also received anti-TNF agents. The authors recommend that patients should be vaccinated before starting methotrexate therapy, but say that an adequate response to vaccination can be achieved after initiation of anti-TNF or low-dose prednisolone treatment.

Jim Casey

Original article Kapetanovic MC et al. (2006) Influence of methotrexate, TNF blockers and prednisolone on antibody responses to pneumococcal polysaccharide vaccine in patients with rheumatoid arthritis. Rheumatology 45: 106-111 